Supporting Information for

\title{
Interplay of Multiexciton Relaxation and Carrier Trapping in Photoluminescent CdS Quantum Dots Prepared in Aqueous Medium
}

\author{
Sharmistha Das, Soumyadipta Rakshit*, Anindya Datta* \\ Department of Chemistry, \\ Indian Institute of Technology Bombay \\ Mumbai 400076, India
}

e-mail: srakshit1987chem@gmail.com (SR), anindya@chem.iitb.ac.in (AD)

Phone: +91 222576 7149, Fax: +91 2225767152 


\section{Table of Contents}

\begin{tabular}{cllc}
\hline Sr. No. & Content & Description & Page \\
\hline 1. & Note SN1 & Transmission Electron Microscopy & S3 \\
\hline 2. & Figure S1 & Transmission electron microscopy of synthesized CdS QDs & S3 \\
\hline 3. & Figure S2 & Second derivative of absorption spectrum & S4 \\
\hline 4. & Table ST1 & $\begin{array}{l}\text { Spectral parameters of Gaussian components of Absorption } \\
\text { Spectrum obtained from Equation 2 }\end{array}$ & S4 \\
\hline 5. & Figure S3 & Excitation Energy dependent PL & S5 \\
\hline 6. & Figure S4 & Wavelength dependent PL transients & S6 \\
\hline 7. & Note SN2 & Calculation of average exciton occupancy $\langle N\rangle$ & S7 \\
\hline 8. & Table ST2 & Estimated average exciton occupancies & S8 \\
\hline 9. & Note SN3 & Calculation of Theoretical Auger Yields & S9 \\
\hline 10. & Figure S5 & Population Analysis & S9 \\
\hline 11. & Table ST3 & Fitting parameters for estimation of biexciton binding energy & S10 \\
\hline 12. & Figure S6 & Analysis of PA Feature & S11 \\
\hline 13. & Figure S7 & Residual Analysis for PA Transient Fitting & S11 \\
\hline 14. & Note SN4 & Calculation of excitation energy dependent PLQY & S12 \\
\hline 15. & Figure S8 & PLQY Analysis & S12 \\
\hline 16. & Note SN5 & Kinetic Model for hot trapping & S13 \\
\hline 17. & Figure S9 & Hot Trapping & S15 \\
\hline 18. & References & &
\end{tabular}




\section{Note SN1: Transmission Electron Microscopy}

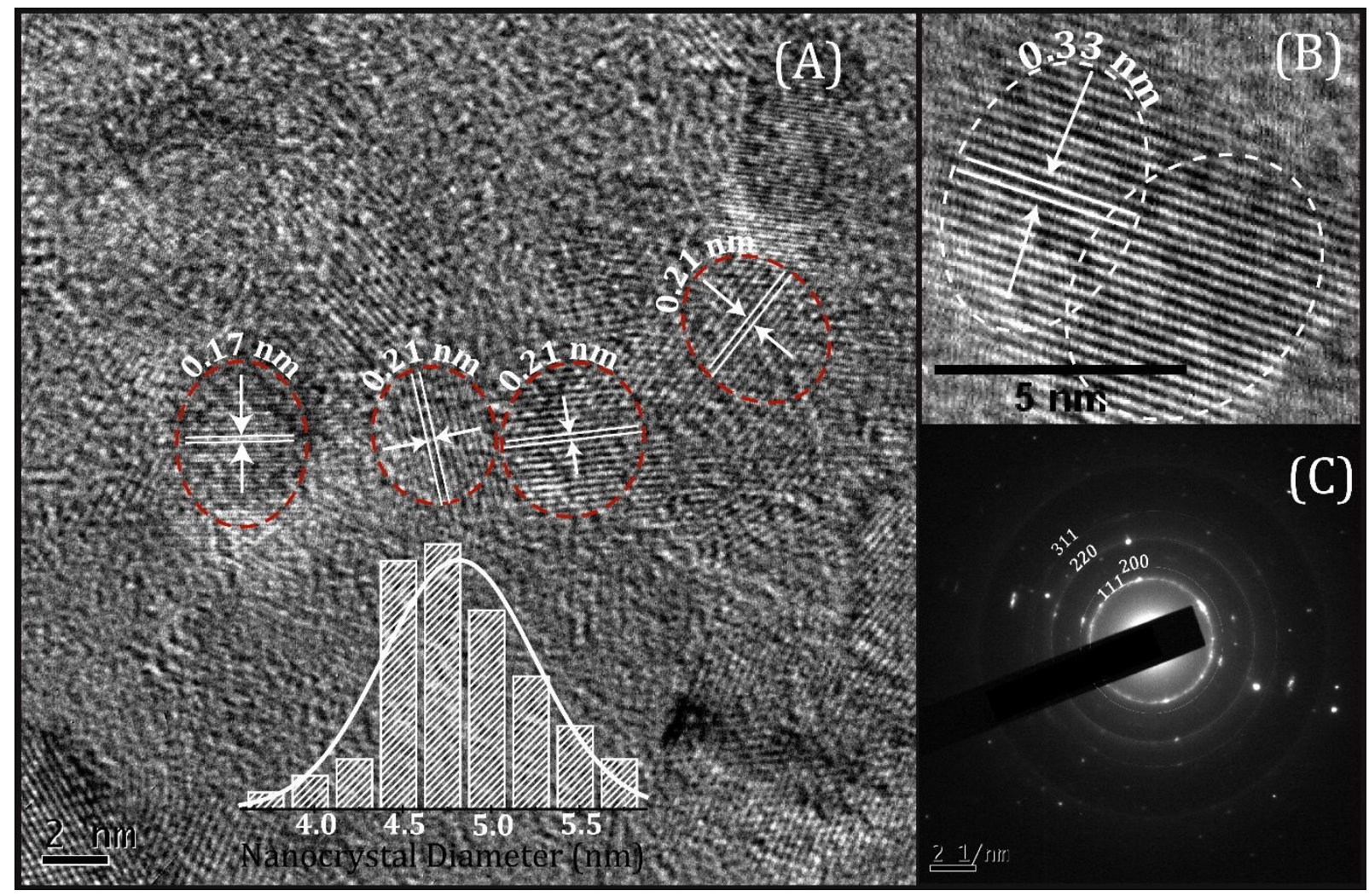

Figure S1. Transmission electron microscopy of synthesized CdS QDs. (a) HRTEM image showing sample crystallinity with interplanar distances and size distribution curve inlaid. (b) Another selection where 111 planes are visible. (c) SAED pattern with the assigned rings indicated.

Well-defined crystal plane striations indicate excellent degree of crystallinity. Interplanar distances of $3.3 \AA, 2.1 \AA$ and $1.7 \AA$ for 111,220 and 311 planes of cubic zinc blende structure are observed, with lattice parameter $\mathrm{a}=5.82 \AA .{ }^{1}$ This is confirmed by analysis of selected area electron diffraction (SAED) patterns. The observed spots and some distinct rings originate due to diffraction from single and multiple crystals respectively, further verifying crystallinity of the samples. Analysis of d-spacings leads to assignment of the rings (Figure S1, C). The structural properties are in keeping with prior reports of room temperature synthesis of $\mathrm{CdS}$ quantum dots of sizes $2.8-5.0 \mathrm{~nm}$ in diameter where a cubic crystal system was observed. ${ }^{1,2}$ The hexagonal wurtzite structure is thermodynamically stable and generally observed in quantum dots obtained using elevated temperatures for synthesis. ${ }^{3,4}$ 


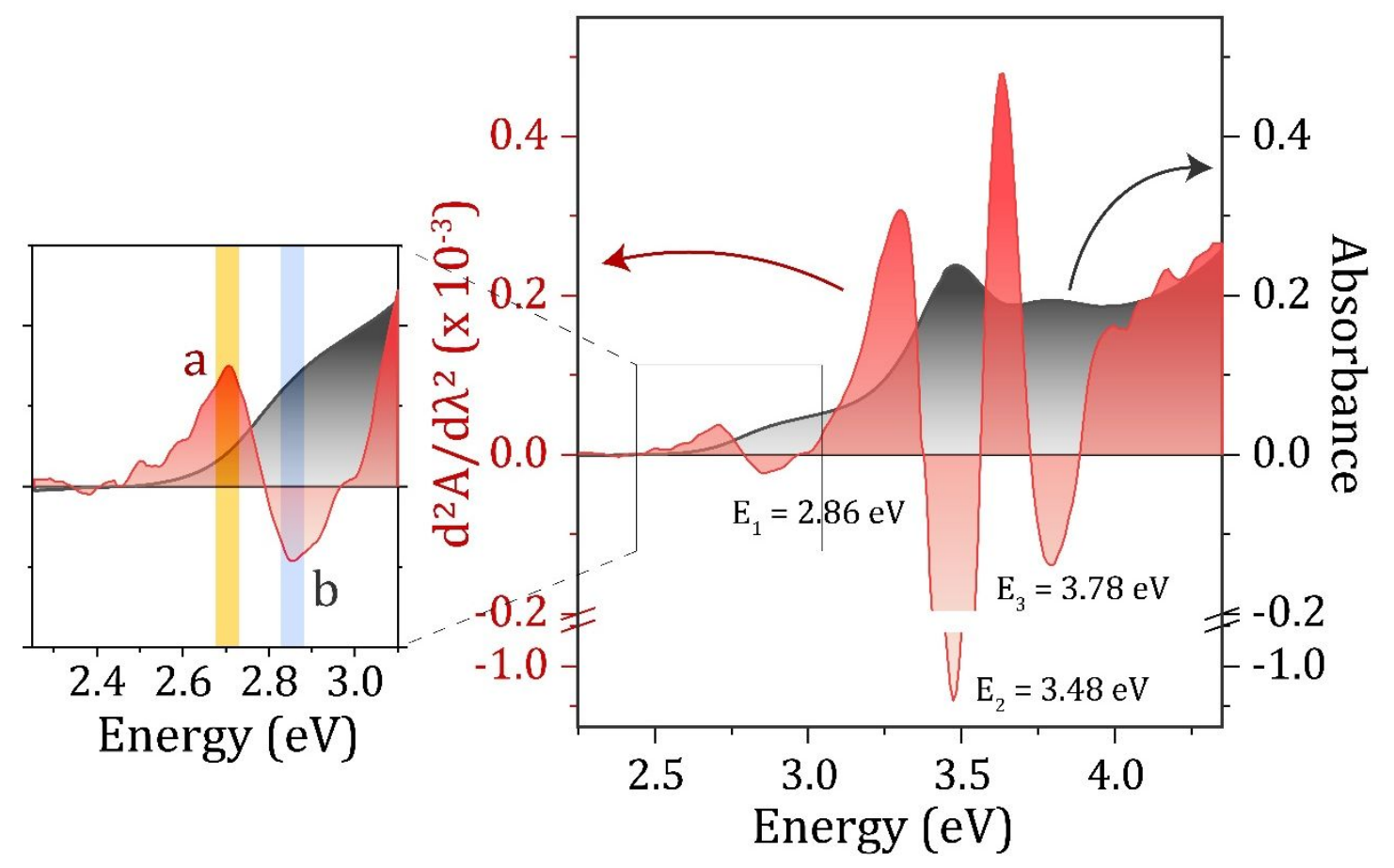

Figure S2. Second Derivative of Absorption Spectrum. Absorbance (black) and its second derivative (red) against wavelength. Minima in the second derivative spectrum correspond to excitonic peaks in the absorption spectrum. The left panel shows the zoomed in absorption onset region as indicated where peak (a) is the absorption onset $(2.72 \mathrm{eV})$ and peak (b) is the $1^{\text {st }}$ excitonic peak $(2.87 \mathrm{eV})$.

Table ST1. Spectral parameters of Gaussian components of Absorption Spectrum obtained from Equation 2

\begin{tabular}{|l|c|c|c|}
\hline & $X_{1}$ & $X_{2}$ & $X_{3}$ \\
\hline Amplitude $a_{i}$ & $0.87 \pm 0.083$ & $3.52 \pm 0.011$ & $1.61 \pm 0.013$ \\
\hline Transition Energy $E_{i}(\mathrm{eV})$ & $2.87 \pm 0.006$ & $3.46 \pm 0.0007$ & $3.76 \pm 0.002$ \\
\hline Linewidth $\sigma_{i}(\mathrm{eV})$ & $0.093 \pm 0.007$ & $0.11 \pm 0.0006$ & $0.12 \pm 0.003$ \\
\hline
\end{tabular}




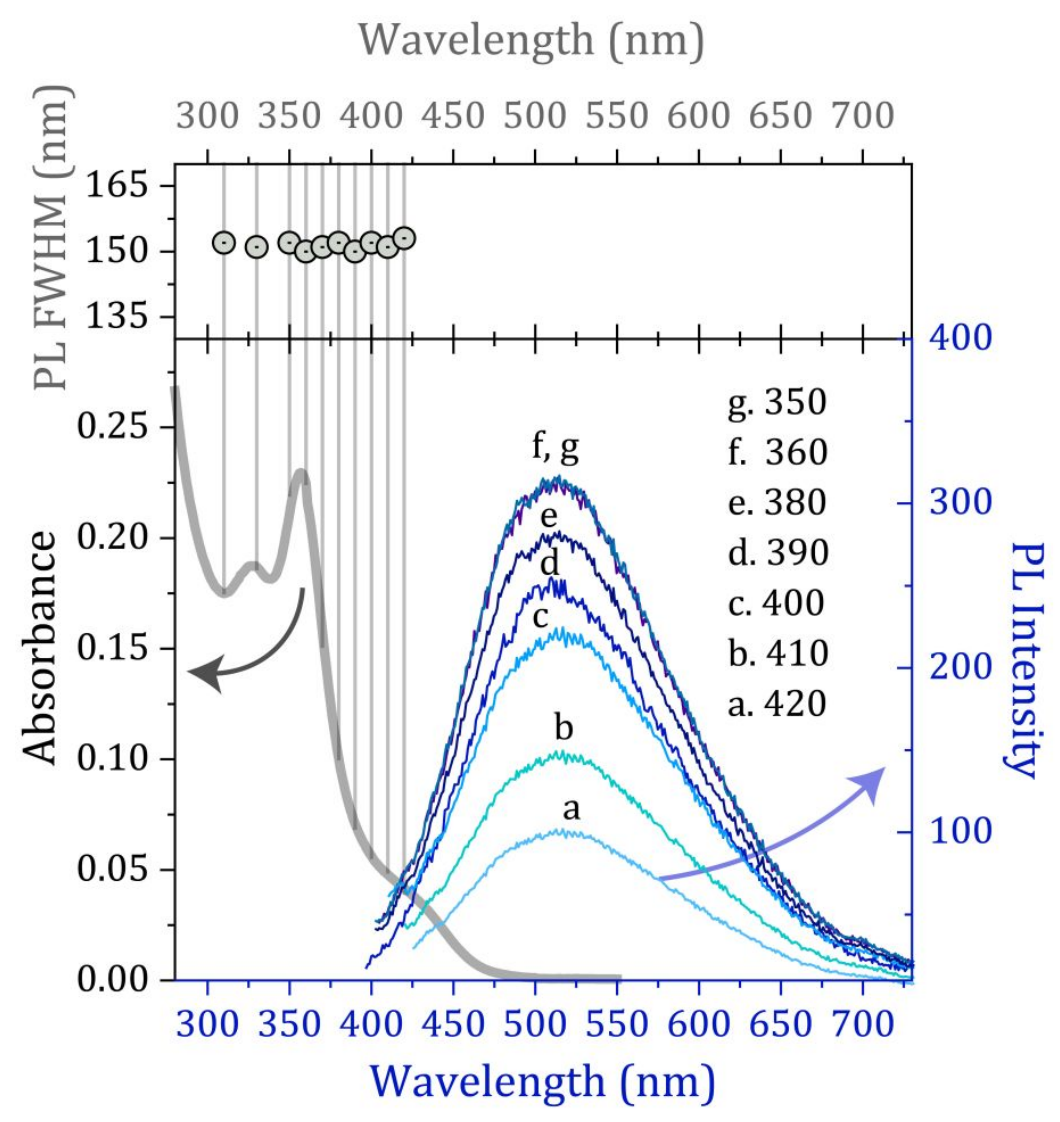

Figure S3. Excitation Energy Dependent PL. (A) Exitation energy dependent PL (blue) and absorption spectra (grey), for perspective on variation of optical density with excitation energy. FWHM for PL spectra are plotted at their correspoding excitation energy in the top pannel. 

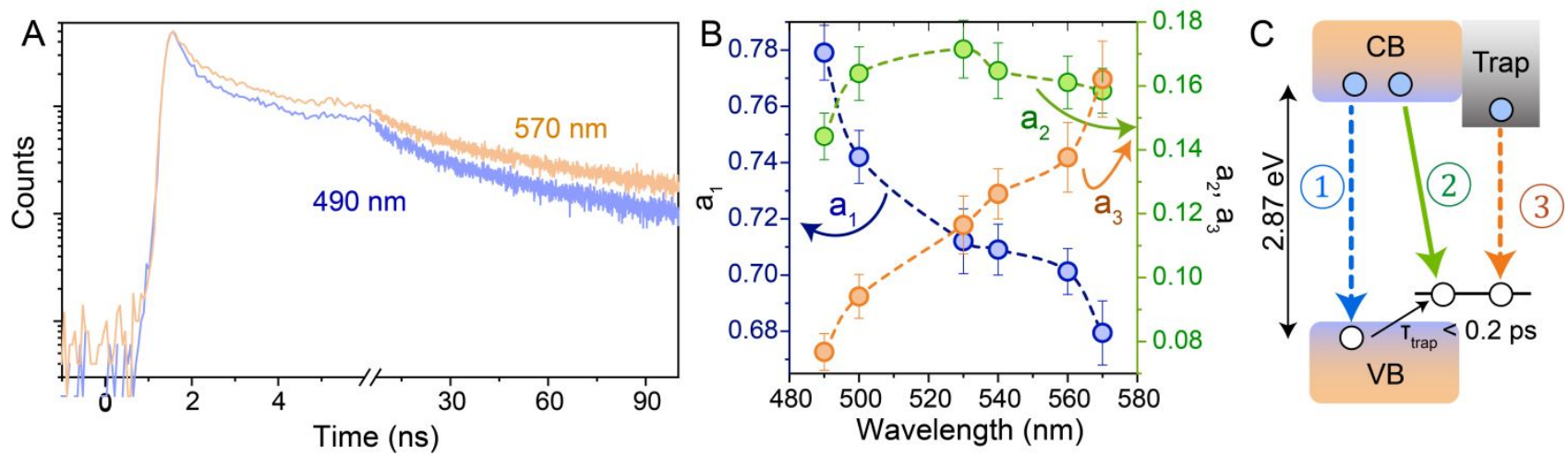

Figure S4. Wavelength dependent PL transients. (A) Nanosecond PL transients CdS QDs monitored at emission wavelengths $490 \mathrm{~nm}$ and $570 \mathrm{~nm}$ (B) PL wavelength dependence of relative amplitudes $a_{1}, a_{2}, a_{3}$ for time constants $\tau_{1}=0.6 n s, \tau_{2}=7.2 n s, \tau_{3}$ $=48 n s$ respectively. (C) Schematic representation of the various exciton recombination pathways $1,2,3$ assigned to $\tau_{1}, \tau_{2}$ and $\tau_{3}$ respectively. 


\section{Note SN2: Calculation of average exciton occupancy $\langle\boldsymbol{N}\rangle$}

\section{(adapted from Reference 6)}

The magnitude of B1 signal arising due to state filling effects is a direct measure of exciton population in semiconductor QDs, assuming a Poisson distribution given by, ${ }^{5}$

$$
P(N)=\langle N\rangle^{N} \frac{e^{-\langle N\rangle}}{N !}
$$

where $\mathrm{P}(\mathrm{N})$ is the probability of having $\mathrm{N}$ excitons in a quantum dot when average number of excitons in the system is $\langle N\rangle$. Normalized 1S bleach intensity is related to $\langle N\rangle$ as ${ }^{5,6}$

$$
\left|\frac{\Delta A}{A}\right|_{1 S}=1-e^{-\langle N\rangle}\left(1+\frac{\langle N\rangle}{2}\right)
$$

Equation 2 represents the average exciton numbers accurately upon band edge excitation. Deviations are observed when bleach intensity is attenuated due to increased contributions from photoinduced absorption signals at high excitation intensities. In case of excitation above band gap, additional contribution to attenuation comes from to hot carrier trapping. Direct trapping of hot carriers, before they cool into the conduction band edge, prepares a population of trapped electrons that never contribute to bleach intensity. To account for both these factors, two parameters $\left\langle N_{0}\right\rangle$ and $\langle N\rangle$ are defined. $\left\langle N_{0}\right\rangle$ is the number of excitons created at zero time:

$$
\left\langle N_{0}\right\rangle=\sigma J_{p}
$$

where $\sigma$ is the absorption cross-section and $j_{p}$ is the photon flux per pump pulse. This value is always approximate due to uncertainties in estimation of both $\sigma$ and $J_{p}$. On the other hand, $\langle N\rangle$ is the average number of excitons determined from the measured $1 \mathrm{~S}$ bleach intensity as defined by equation 2. To circumvent the errors inducted due to bleach attenuation by increased contributions of photoinduced absorptions at high excitation intensities, $\langle N\rangle$ is estimated using equation 2 at lowest excitation intensity used with negligible photoinduced absorption. Then equations 2 and 3 are combined to arrive at equation 4 to determine $\langle N\rangle$ at higher excitation intensities.

$$
\frac{\langle N\rangle_{1}}{E_{1}}=\frac{\langle N\rangle_{2}}{E_{2}}
$$


$E_{i}$ is energy per pulse of excitation beam and $\langle N\rangle_{i}$ the corresponding average exciton number (Table ST2). $\langle N\rangle$ is used in further labelling and analysis of data acquired with different pump fluences as it is a reasonably accurate estimation of $1 \mathrm{~S}$ state population.

Table ST2. Estimated average exciton occupancies.

\begin{tabular}{|c|c|}
\hline Energy $(\mu \mathrm{J})$ & $\langle N\rangle$ \\
\hline 1.2 & 0.59 \\
1.8 & 0.88 \\
\hline 3.0 & 1.46 \\
\hline 4.0 & 1.94 \\
\hline
\end{tabular}




\section{Note SN3. Estimation of Theoretical Auger Yields}
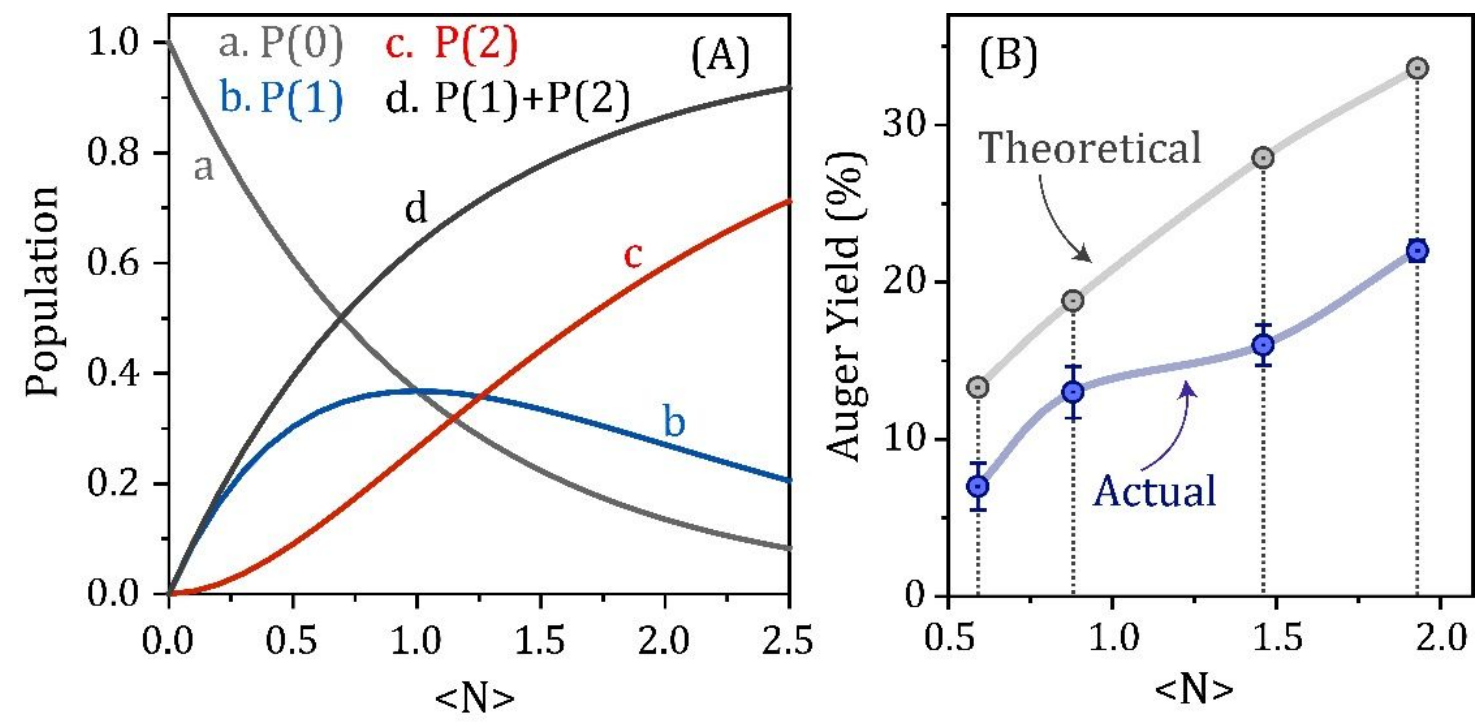

Figure S5. Population Analysis. $\langle N\rangle$-dependence of (A) calculated populations for QDs with a. unexcited population $P(0)$, b. single excitons $P(1)$ c. biexcitons $P(2)$ and d. total excited population $P(1)+P(2)$. (B) contributions of experimental and theoretical Auger recombination to $\mathrm{B} 1$

Expected contribution of biexciton recombination, to the overall excitonic bleach transient, is estimated by an elementary Poisson distribution statistics analysis, proposed by Weiss and co-workers. ${ }^{7}$ The number of excitons are limited to 2 in the calculations to account for the inherent double degeneracy of the $1 S_{e}$ state. In other words, even if more than 2 photons are absorbed by a quantum dot, only biexcitons contribute to the excitonic bleach transients. Populations $P(N)$ of QDs having 0 or 1 exciton, are calculated using equation 1 with $\mathrm{N}=$ 0,1 and $\langle N\rangle$ as per excitation intensities used. $P(2)$ is then calculated as $P(2)=1-P(0)$ $-P(1)$ (Figure S3A). Theoretical Auger recombination can then the calculated as,

$$
\text { Theoretical Auger } T_{A R}(\%)=\frac{1}{2 P(1)+P(2)}
$$

The factor of $1 / 2$ accounts for the fact that in a QD with two excitons, biexcitonic auger recombination can occur only once. This value thus quantifies the maximum possible contribution of Auger recombination to B1 signal (Table 1). Deviations between theoretical and experimental values, attributed to trapping processes. increase at higher values of $\langle N\rangle$ (Figure 3B) due to enhanced trapping rates with increasing pump fluence. 
Table ST3. Estimation of biexciton binding energy.

\begin{tabular}{|c|c|c|c|}
\hline$\Delta t=$ & $0.20 \mathrm{ps}$ & $0.28 \mathrm{ps}$ & $0.35 \mathrm{ps}$ \\
\hline$a_{1}$ & $-0.087 \pm 0.0046$ & $-0.054 \pm 0.0012$ & $-0.057 \pm 0.0045$ \\
\hline$E_{1}$ & $2.87 \pm 0.0014$ & $2.86 \pm 0.0012$ & $2.87 \pm 0.0015$ \\
\hline$\sigma$ & $0.29 \pm 0.0046$ & $0.26 \pm 0.004$ & $0.24 \pm 0.0048$ \\
\hline$\Delta_{x x}$ & $0.023 \pm 9.8 \mathrm{E}-5$ & $0.029 \pm 0.0002$ & $0.027 \pm 0.0002$ \\
\hline$a_{2}$ & $-0.084 \pm 2.05 \mathrm{E}-5$ & $-0.045 \pm 0.0013$ & $-0.046 \pm 0.0025$ \\
\hline
\end{tabular}

Average Binding energy: $26 \mathrm{meV}$ 

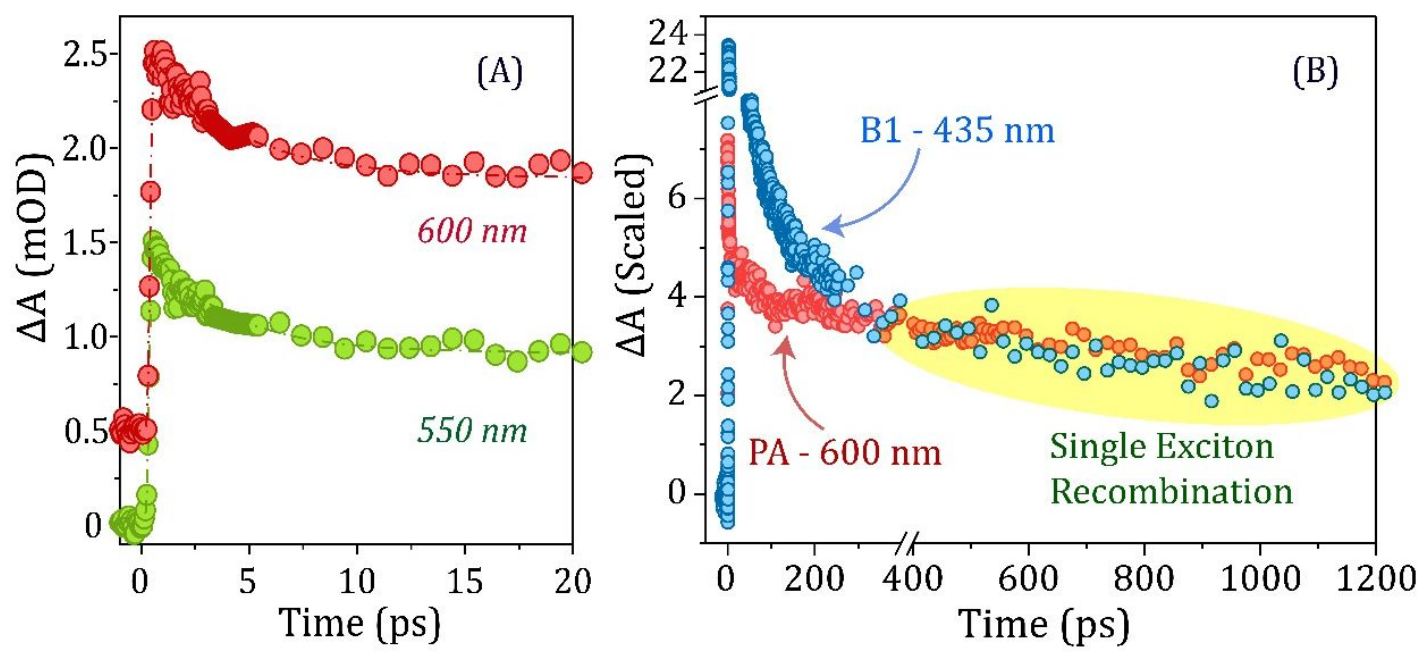

Figure S6. Analysis of PA Feature. (A) PA transients at $\lambda_{\text {probe }}=550 \mathrm{~nm} \& 600 \mathrm{~nm}$, vertically translated for better clarity. The wavelength independence of shelf feature kinetics is evident. Similar trends are observed at all wavelengths in this region for all excitation fluences. (B) Comparing B1 and PA signal transients with $\lambda_{\text {probe }}=435 \mathrm{~nm} \& 600 \mathrm{~nm}$ respectively measured at an excitation fluence corresponding to $\langle N\rangle=0.88$. The transients are scaled to overlap at long delay times for comparison.

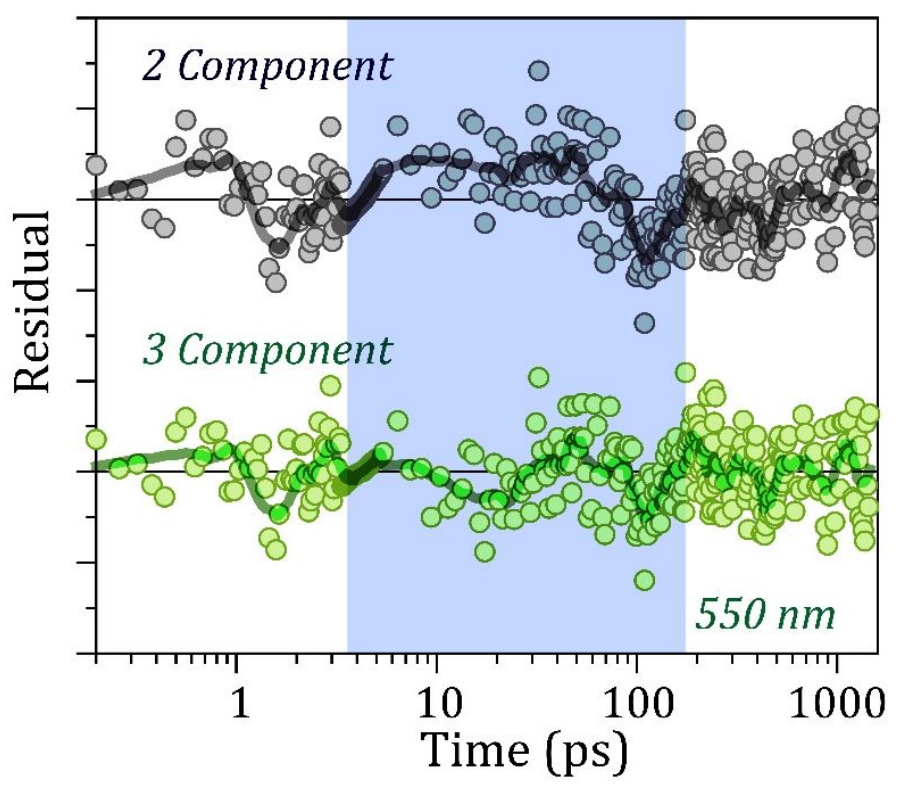

Figure S7. Residual Analysis. Residuals for fitting of PA transient at $\lambda_{\text {probe }}=550 \mathrm{~nm}$ and $\langle N\rangle$ $=0.88$ with 2 components (grey, top) and 3 components (green bottom). Significant improvement of residuals observed in highlighted region (blue, centre). Similar observations are made at all probe wavelengths in the PA feature and at all $\langle N\rangle$ values. 
Note SN4. Calculation of excitation energy dependent PLQY
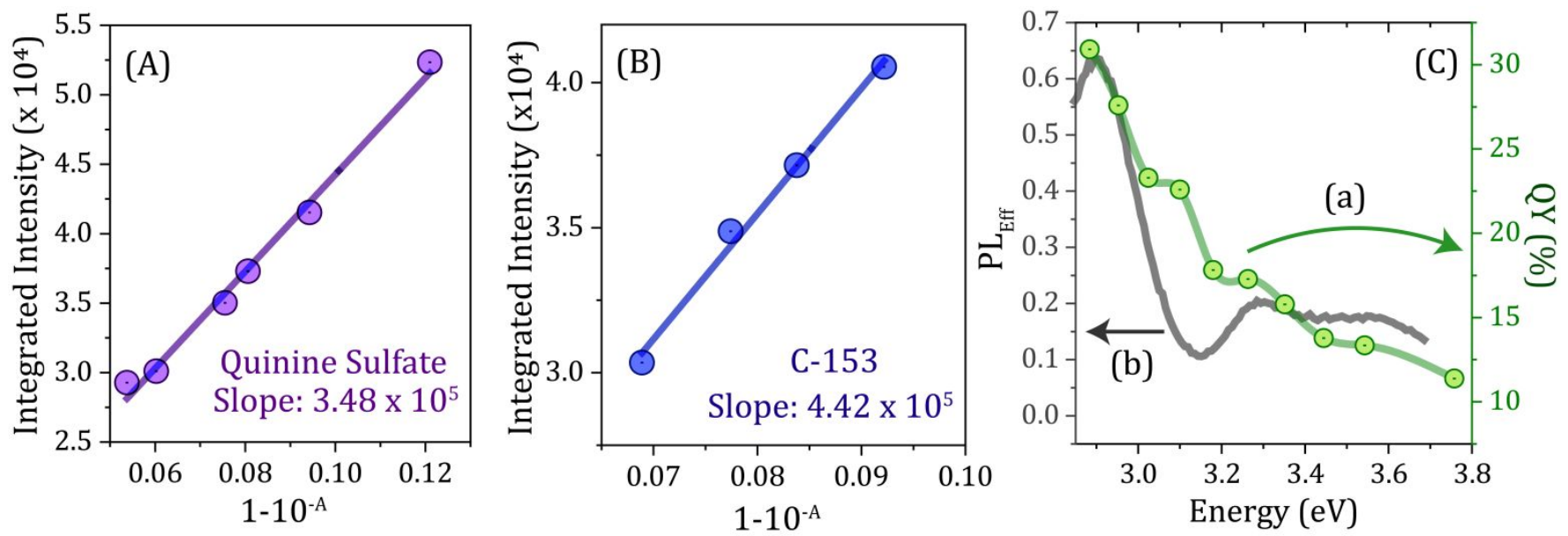

Figure S8. PLQY Analysis. Integrated Intensity vs absorbance plots of (A) Quinine Sulfate and (B) C-153 used as reference standards for calculation of QY of CdS QDs at various excitation energies. (C) Comparison of QY (curve a) and $\mathrm{PL}_{\mathrm{Eff}}$ (curve $\mathrm{b}$ ) calculated from PLE spectra.

Photoluminescence quantum yield (PLQY) of the synthesized CdS quantum dots are calculated using standard protocols of relative quantum yield calculation, as defined by equation 6 below.

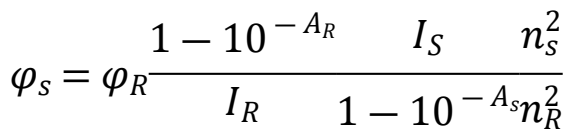

$\varphi, I, A$ and $n$ are for quantum yield, integrated intensity, optical density and refractive index of the solutions used, respectively. The subscript ' $S$ ' and ' $R$ ' represent sample and reference in the above equation. Optical density values are less than $\sim 0.08$ in the entire range covered. Quinine Sulfate $(\varphi=0.58)^{8}$ in $0.1 \mathrm{M} \mathrm{H}_{2} \mathrm{SO}_{4}$ and Coumarin-153 in methanol $(\varphi=0.54)^{9}$ are used as reference dyes to accurately cover the entire range of excitation energies. Additionally, photoaction spectra, i.e. PL excitation spectra normalized with absorbance at each wavelength (equation 7), is constructed in order obtain an accurate correction for the number of photons absorbed versus emitted. Hence this is an approximate measure for variation of quantum yield with excitation energy.

$$
P L_{e f f}=\frac{P L E(E)}{1-10^{-A(E)}}
$$




\section{Note SN5. Kinetic Model for Hot Electron Trapping}

B1 monitors population dynamics of conduction band electrons and so, depends on the electron population at the conduction band minimum (CBM). Its growth reflects hot electron relaxation to the CBM. Faster carrier cooling times in presence of traps or electron/hole acceptors are indicators of hot carrier trapping or transfer. ${ }^{10-12}$ The following kinetic equations describe the population dynamics in the conduction band:

$$
\begin{aligned}
& \frac{d n_{1}}{d t}=-\left(k_{10}+k_{h}\right) n_{1}(t) \\
& \frac{d n_{0}}{d t}=k_{10} n_{1}(t)-k_{c} n_{0}(t)
\end{aligned}
$$



where, $n_{1}$ is the population of the initially excited state, $n_{0}$ the population of electrons at conduction band minima, $k_{10}$ the intraband relaxation rate, $k_{h}$ the trapping rate for hot electrons, and $k_{c}$ the trapping rate of cold electrons. Solution of the above equations yields:

$$
n_{0}(t)=\frac{k_{10} n_{1}(0)}{k_{10}+k_{h}-k_{c}}\left(e^{-k_{c} t}-e^{-\left(k_{10}+k_{h}\right) t}\right) \otimes I R F
$$

The fitting procedure includes deconvolution of the IRF (equation 10) and added long components to account for long time kinetics. The first exponential term corresponds to cold electron trapping while the second term is the bleach rise time which is modulated by hot trapping rates in addition to intraband relaxation. Furthermore, the pre-exponential factor which determines the bleach magnitude also has hot trapping rate in denominator, thus signifying enhanced trapping of hot carriers amounts to greater attenuation of bleach intensities. The simple model described above fits the measured kinetics remarkably well as observed from the fitting curves (Figure S8, vide infra). 

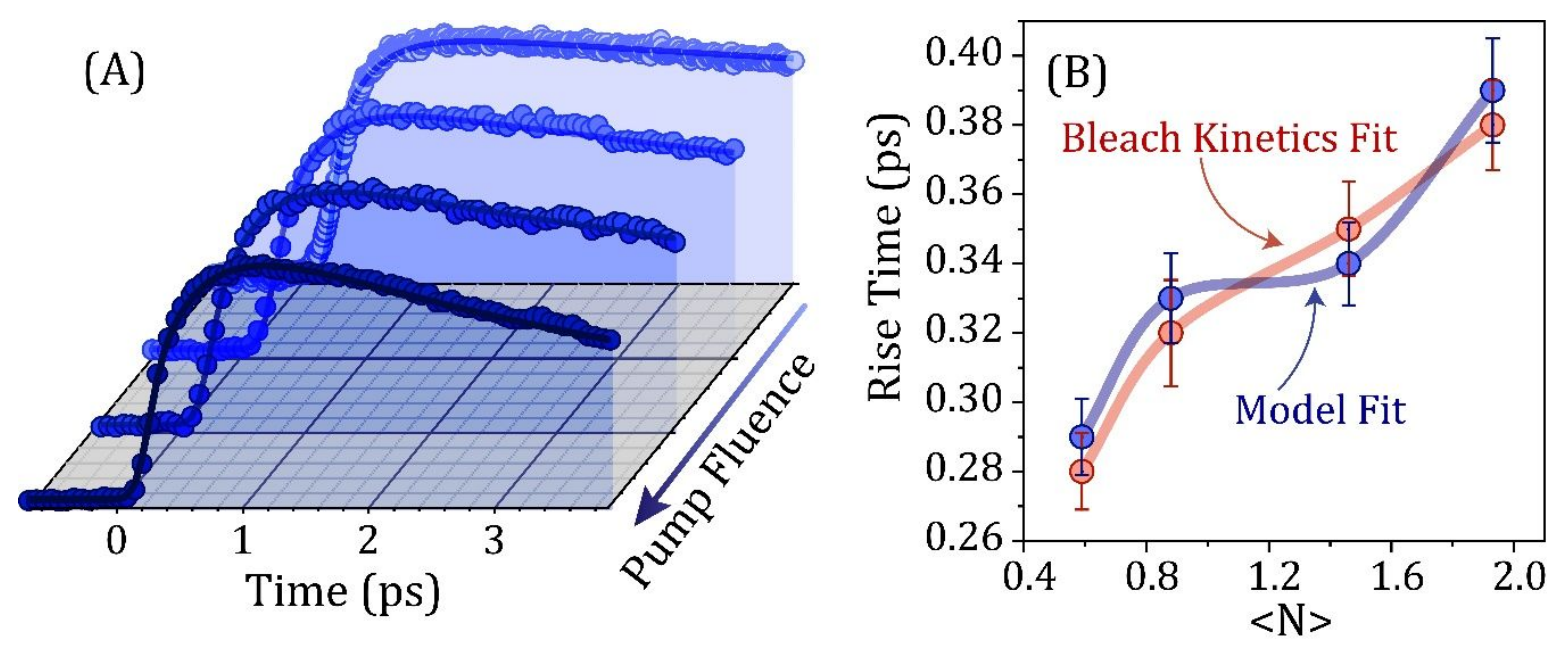

Figure S9. Kinetic Model of Hot Trapping. (A) Bleach transients $\left(\lambda_{\text {probe }}=435 \mathrm{~nm}\right)$ at different pump fluences at initial delay times (upto $4 \mathrm{ps}$ ). The solid lines are the fits to the data points with the kinetic model. (B) Graphical comparison of rise times obtained with multiexponential fit (red) and kinetic model (blue) at various excitation intensities. 


\section{References}

(1) Unni, C.; Philip, D.; Gopchandran, K. G. Studies on Optical Absorption and Photoluminescence of Thioglycerol-Stabilized CdS Quantum Dots. Spectrochim. Acta - Part A Mol. Biomol. Spectrosc. 2008, 71 (4), 1402-1407.

(2) Pal, M.; Mathews, N. R.; Santiago, P.; Mathew, X. A Facile One-Pot Synthesis of Highly Luminescent CdS Nanoparticles Using Thioglycerol as Capping Agent. $J$. Nanoparticle Res. 2012, 14, 916.

(3) Vossmeyer, T.; Reck, G.; Katsikas, L.; Haupt, E. T. K.; Schulz, B.; Weller, H. A "Double-Diamond Superlattice" Built up of Cd17S4(SCH2CH2OH)26 Clusters. Science (80-. ). 1995, 267, 1476-1479.

(4) Zhong, X.; Liu, S.; Zhang, Z.; Li, L.; Wei, Z.; Knoll, W. Synthesis of High-Quality CdS, ZnS5 and ZnxCd1 - XS Nanocrystals Using Metal Salts and Elemental Sulfur. $J$. Mater. Chem. 2004, 14, 2790-2794. https://doi.org/10.1039/b407556k.

(5) Klimov, V. I. Spectral and Dynamical Properties of Multiexcitons in Semiconductor Nanocrystals. Annu. Rev. Phys. Chem. 2007, 58, 635-673.

(6) Tyagi, P.; Cooney, R. R.; Sewall, S. L.; Sagar, D. M.; Saari, J. I.; Kambhampati, P. Controlling Piezoelectric Response in Semiconductor Quantum Dots via Impulsive Charge Localization. Nano Lett. 2010, 10, 3062-3067.

(7) Lian, S.; Christensen, J. A.; Kodaimati, M. S.; Rogers, C. R.; Wasielewski, M. R.; Weiss, E. A. Oxidation of a Molecule by the Biexcitonic State of a CdS Quantum Dot. J. Phys. Chem. C 2019, 123, 5923-5930.

(8) Lakowicz, J. R. Principles of Fluorescence Spectroscopy Principles of Fluorescence Spectroscopy; 2006.

(9) Kubin, R. F.; Fletcher, A. N. The Effect of Oxygen on the Fluorescence Quantum Yields of Some Coumarin Dyes in Ethanol. Chem. Phys. Lett. 1983, 99, 49-52.

(10) Smith, C. T.; Leontiadou, M. A.; Page, R.; O’Brien, P.; Binks, D. J. Ultrafast Charge Dynamics in Trap-Free and Surface-Trapping Colloidal Quantum Dots. Adv. Sci. 2015, $2,1-7$.

(11) De, A.; Das, S.; Samanta, A. Hot Hole Transfer Dynamics from CsPbBr3Perovskite Nanocrystals. ACS Energy Lett. 2020, 5, 2246-2252.

(12) Mondal, N.; De, A.; Samanta, A. All-Inorganic Perovskite Nanocrystal Assisted Extraction of Hot Electrons and Biexcitons from Photoexcited CdTe Quantum Dots. Nanoscale 2018, 10 (2), 639-645. 